\title{
On the Renormalization of CPT/Lorentz Violating QED in Curved Space
}

\author{
Guilherme de Berredo-Peixoto $^{a, b}{ }^{1}, \quad$ Ilya L. Shapiro ${ }^{b}$ \\ a. Departamento de Engenharia Rural, CAV, Universidade do Estado de Santa Catarina, \\ Lages, 88520-000, SC, Brazil \\ b. Departamento de Física - ICE, Universidade Federal de Juiz de Fora, \\ Juiz de Fora, 36036-330, MG, Brazil
}

\begin{abstract}
We consider the one-loop renormalization of QED in curved space-time with additional Lorentz and/or CPT breaking terms. The renormalization group equations in the vacuum sector are derived. In the special case of Minkowski metric and with constant Lorentz and CPT breaking terms these equations reduce to the ones obtained earlier by other authors. The necessary form of the vacuum counterterms indicate possible violations of the space or time homogeneity or space isotropy in the gravitational phenomena. However, the necessity of the phenomenologically most interesting terms such as linear in the space-time curvature or torsion, is related to the non-constant nature of the dimensionless Lorentz and CPT breaking parameters.
\end{abstract}

Keywords: CPT and Lorentz violation, Curved space, Renormalization. PACS: 04.62.+v, 12.90.+b.

\section{Introduction}

One of the most perspective windows into the Planck-scale physics is due to the possible violations of the fundamental (Lorentz and/or CPT) symmetries [1]. According to the existing theoretical investigations [2], it might happen that, due to the string scale quantum effects, the vacuum is not Lorentz invariant. This means that in our part of the Universe there is some fixed external field or fields which define a preferable direction in the space-time. Similar vacuum effects may be responsible for the CPT violations. The conventional approach for the study of the possible Lorentz and CPT violations is to consider the most general form for these violations and then look for their phenomenological consequences. The most promising type of experiments here belong to the area of atomic physics [3], but there are also interesting options in the area of high energy and solid state physics [4], neutrinos experiments [5], gravitation [6] and cosmology [7] (see further references therein).

From the quantum field theory viewpoint, the presence of additional external fields means that the changes may occur not only at the tree level but also in the loop corrections. The investigation of the loop correction in CPT and Lorentz violated QED has been started recently in the work [8], where the renormalization-group $\beta$-functions have been derived and the corresponding quantum

\footnotetext{
${ }^{1}$ E-mail address: guilherme@fisica.ufjf.br

${ }^{2}$ Also at Tomsk State Pedagogical University, Russia. E-mail address: shapiro@fisica.ufjf.br
} 
effects were indicated. One of the most important aspects of quantum consideration is that it enables one to elaborate the criteria of consistency for the theory. These criteria concern the minimal necessary set of the terms in the classical action which are usually required by renormalizability and unitarity of the theory and also the magnitudes of the new parameters in the actions, which should be consistent with the renormalization group running caused by the loop effects. An example of the theory where these criteria were very well investigated is the quantum field theory in curved spacetime. In this case the consistency is achieved by introducing the so-called vacuum action and the non-minimal interaction terms for the scalar fields. The most remarkable fact is that the vacuum action must include, along with the Einstein-Hilbert term, also the cosmological constant and the full set of local fourth derivative terms (see, e.g. 10 for the introduction and further references). This aspect of the theory leads to important consequences for the gravitational phenomenology and, in particular, is relevant for a better understanding of the Cosmological Constant Problem [11.

Indeed, one can consider the situation with the Lorentz and CPT violating terms in QED (or any other quantum field theory) from a similar viewpoint. Introducing new terms in the matter sector one meets, at the quantum level, the necessity to extend the classical action of vacuum by introducing the full set of the new terms depending on Lorentz and CPT violating parameters. The form of the necessary new terms may be established on the basis of general considerations using dimension and covariance. However, only practical one-loop calculations may indicate which of those terms are the most important ones. Let us remember that, due to the weakness of the Lorentz and CPT violating terms, the higher loop vacuum effects are definitely very small. Therefore the most important terms are those which will emerge in the one-loop counterterms. Hence the correct strategy in investigating the possible role of Lorentz and CPT violating parameters in the gravitational sector is to start by deriving the one-loop divergences for the quantum field theory on curved background, including the Lorentz and CPT violating terms in the matter section. The results of such calculation can be used as a hint, indicating the most important new terms to be implemented into the gravitational action. At the next stage one has to look for the possible phenomenological manifestation of these new terms.

In this paper we report on the particular results of the one-loop calculations in the Lorentz and CPT violating QED in curved space-time. This calculation helps us to identify the most interesting gravitational terms, that may be a good starting point for the phenomenological analysis.

The paper is organized as follows. In section 2 we consider the Lorentz and CPT violating QED in curved space-time and derive the most relevant one-loop counterterms. Our results are less general than the ones of the previous calculation in flat space-time [8], because we set some of the new parameters to zero. The inclusion of these parameters leads to enormous increase of the size of the calculational work and we postpone this calculation for the future. On the other hand, we perform a more general calculation that the one of $[8$, because we do not request nor that the Lorentz and CPT violating parameters are constants ${ }^{3}$, neither the flatness of the space-time. In this way we achieve, in section 3 , the form of the qualitatively new terms in the gravitational action and consider the renormalization group for (some of) the corresponding parameters. The

\footnotetext{
${ }^{3}$ The possibility of the space-time dependent Lorentz-violating parameters has been considered in 9 .
} 
section 4 includes a general discussion of their possible physical effects and in section 5 we draw our conclusions.

\section{Derivation of the one-loop divergences}

Our starting point is the action describing an extended version of QED with Lorentz and CPT symmetry breaking terms, minimally coupled to gravitation

$$
\begin{aligned}
S & =\int d^{4} x \sqrt{-g}\left\{\frac{i}{2} \bar{\psi} \Gamma^{\mu} D_{\mu} \psi-\frac{i}{2} D_{\mu}^{\star} \bar{\psi} \Gamma^{\mu} \psi-\bar{\psi} M \psi-\frac{1}{4} F_{\mu \nu} F^{\mu \nu}\right. \\
& \left.-\frac{1}{4}\left(k_{F}\right)_{\mu \nu \alpha \beta} F^{\mu \nu} F^{\alpha \beta}+\frac{1}{2}\left(k_{A F}\right)^{\rho} \varepsilon_{\rho \lambda \mu \nu} A^{\lambda} F^{\mu \nu}\right\} .
\end{aligned}
$$

Here

$$
D_{\mu}=\nabla_{\mu}+i q A_{\mu} ; \quad D_{\mu}^{\star}=\nabla_{\mu}-i q A_{\mu} ; \quad \Gamma^{\nu}=\gamma^{\nu}+\Gamma_{1}^{\nu} ; \quad M=m+M_{1}
$$

the operator $\nabla_{\mu}$ is the covariant derivative, $F_{\mu \nu}=\nabla_{\mu} A_{\nu}-\nabla_{\nu} A_{\mu}$ and the quantities $\Gamma_{1}^{\nu}$ and $M_{1}$ are given by

$$
\begin{aligned}
& \Gamma_{1}^{\nu}=c^{\mu \nu} \gamma_{\mu}+d^{\mu \nu} \gamma_{5} \gamma_{\mu}+e^{\nu}+i f^{\nu} \gamma_{5}+\frac{1}{2} g^{\lambda \mu \nu} \sigma_{\lambda \mu}, \\
& M_{1}=a_{\mu} \gamma^{\mu}+b_{\mu} \gamma_{5} \gamma^{\mu}+i m_{5} \gamma_{5}+\frac{1}{2} H_{\mu \nu} \sigma^{\mu \nu} .
\end{aligned}
$$

Along with the parameters $\left(k_{F}\right)_{\mu \nu \alpha \beta}$ and $\left(k_{A F}\right)^{\rho}$, the quantities $a_{\mu}, b_{\mu}, m_{5}, c^{\mu \nu}, d^{\mu \nu}, e^{\mu}, f^{\mu}, g^{\lambda \mu \nu}$ and $H_{\mu \nu}$ are CPT and/or Lorentz violating parameters. An extensive discussion of the possible origin of these parameters and also their numerous phenomenological implications can be found in [1, 2] and we will not consider these aspects here. Let us only notice that the quantities constituting $\Gamma_{1}^{\nu}$ and the parameter $\left(k_{F}\right)_{\mu \nu \alpha \beta}$ are dimensionless and all other parameters have non-zero dimensions which can be easily found from eq. (11).

Our purpose is to investigate the one-loop renormalization in the vacuum sector, depending on the metric and on the set of the CPT and/or Lorentz violating parameters. As usual, we assume that these new fields are small, therefore our calculation can be restricted to the first order in the Lorentz and CPT violating parameters. At the same time, we do not see why these parameters must be constants and hence we will let them being local functions of the space-time coordinates. Indeed, the space and time derivatives of these parameters should be small, such that the linear approximation in the new parameters and their derivatives remain sufficient.

In order to investigate the one-loop renormalization of the Lorentz/CPT breaking coefficients, we consider the background field method splitting,

$$
\psi \rightarrow \psi+\eta ; \quad \bar{\psi} \rightarrow \bar{\psi}+\bar{\eta} ; \quad A_{\mu} \rightarrow A_{\mu}+B_{\mu}
$$

where $\eta, \bar{\eta}$ and $B_{\mu}$ are the quantum fields, and calculate the complete one-loop divergences of theory (1), given by ${ }^{4}$

$$
\Gamma_{\text {div }}^{(1)}=\left.\frac{i}{2} \mathrm{~s} \operatorname{Tr} \ln \hat{\mathcal{H}}\right|_{\text {div }} .
$$

\footnotetext{
${ }^{4}$ The definition of the operation of supertrace sTr is given below, after the eq. (17).
} 
where the operator $\hat{\mathcal{H}}$ is the operator of the bilinear in quantum fields part of the action together with the gauge-fixing term. Let us introduce this term in the form

$$
S_{g f}=\frac{1}{2 \alpha} \int d^{4} x \sqrt{-g}\left(\nabla_{\mu} B^{\mu}\right)^{2},
$$

where $\alpha$ is an arbitrary parameter of the gauge fixing. Let us notice that, with this choice of the gauge-fixing, the corresponding Faddeev-Popov ghosts contribute only to the vacuum (metricdependent) sector of the theory and these contributions do not depend on the new Lorentz breaking parameters of the theory. Therefore one can safely disregard the contribution of the ghosts except when we consider these terms in eq. (20). The use of the background field method (see [10] for the introduction and further references) guarantees the gauge invariance of the quantum corrections.

For practical calculations we choose the value of $\alpha=-1$, such that without the Lorentz breaking parameters, the bilinear form of the action has the most simple minimal form, with second derivatives forming the $\square=g^{\mu \nu} \nabla_{\mu} \nabla_{\nu}$ combination (see, for example, the expression (9) below, in the case $\hat{\mathcal{K}}^{\rho \lambda}=0$ ). The natural question is whether the quantum corrections may depend on the choice of parameter $\alpha$. As far as we are interested in the one-loop divergences and related objects like $\beta$-functions, the well known general theorems [12, 13, tell us that the gauge fixing dependence should disappear on the classical mass-shell. As a result, only the renormalization of the wave function for the fermion may depend on the choice of $\alpha$, while the $\beta$-functions for all parameters (including the Lorentz-violating ones) are not affected by this dependence.

After performing the bilinear expansion, it proves useful to make a change of variables

$$
\eta=-\frac{i}{2}\left(\gamma^{\mu} \nabla_{\mu}-i m\right) \chi
$$

The corresponding Jacobian depends only on the metric and can be evaluated using the known algorithms (see, e.g. [10]). After the mentioned change of quantum variables the bilinear part of the total action acquires the form

$$
S=\frac{1}{2} \int d^{4} x \sqrt{-g}\left(\begin{array}{c|c}
B_{\mu} & \bar{\eta}
\end{array}\right) \hat{\mathcal{H}}\left(\begin{array}{c}
B_{\nu} \\
\chi
\end{array}\right) .
$$

Let us notice that the above expression has an unusual form, making practical calculations rather difficult. Due to the presence of the massless parameters in $\Gamma_{1}^{\nu}$ and also $\left(k_{F}\right)_{\mu \nu \alpha \beta}$ in the action (1), the differential operator $\hat{\mathcal{H}}$ has a general non-minimal structure

$$
\hat{\mathcal{H}}=\hat{\mathcal{K}}^{\rho \lambda} \nabla_{\rho} \nabla_{\lambda}+\hat{\mathbf{1}} \square+2\left(\hat{h}_{0}^{\rho}+\hat{L}^{\rho}\right) \nabla_{\rho}+\left(\hat{\Pi}_{0}+\hat{\mathcal{M}}\right),
$$

where we have separated the conventional terms and the new, (unusual) ones. In particular,

$$
\hat{\mathbf{1}}=\left(\begin{array}{c|c}
g^{\mu \nu} & 0 \\
0 & \mid
\end{array}\right)
$$

with $I$ being the identity operator in the fermionic sector, $\hat{h}_{0}^{\rho}$ and $\hat{\Pi}_{0}$ are those parts of the corresponding expressions (matrices), which are independent on the Lorentz violating coefficients. These formulas simply correspond to the pure QED theory in curved space-time. Their explicit form is

$$
\hat{h}_{0}^{\rho}=\left(\begin{array}{cc}
0 & \frac{i}{2} q \bar{\psi} \gamma^{\mu} \gamma^{\rho} \\
0 & \frac{i}{2} q \gamma^{\alpha} \gamma^{\rho} A_{\alpha}
\end{array}\right), \quad \hat{\Pi}_{0}=\left(\begin{array}{cc}
-R^{\mu \nu} & m q \bar{\psi} \gamma^{\mu} \\
-2 q \gamma^{\nu} \psi & m^{2} I-\frac{I}{4} R+m q \gamma^{\alpha} A_{\alpha}
\end{array}\right)
$$


Furthermore, the matrices $\hat{\mathcal{K}}^{\rho \lambda}, \hat{L}^{\rho}$ and $\hat{\mathcal{M}}$ are linear on the Lorentz violating coefficients,

$$
\begin{gathered}
\hat{\mathcal{K}}^{\rho \lambda}=\left(\begin{array}{cc}
-2 k_{F}^{\mu(\rho \lambda) \nu} & 0 \\
0 & \Gamma_{1}^{(\rho} \gamma^{\lambda)}
\end{array}\right), \\
\hat{L}^{\rho}=\left(\begin{array}{cc}
\nabla_{\tau} k_{F}^{\tau \mu \rho \nu}-\left(k_{A F}\right)_{\tau} \varepsilon^{\tau \mu \nu \rho} & \frac{i}{2} q \bar{\psi} \Gamma_{1}^{\mu} \gamma^{\rho} \\
0 & \frac{i}{2} q \Gamma_{1}^{\alpha} \gamma^{\rho} A_{\alpha}-\frac{i}{2} m \Gamma_{1}^{\rho}+\frac{i}{2} M_{1} \gamma^{\rho}+\frac{1}{4} \nabla_{\mu} \Gamma_{1}^{\mu} \gamma^{\rho}
\end{array}\right)
\end{gathered}
$$

and

$$
\hat{\mathcal{M}}=\left(\begin{array}{cc}
\nabla_{\rho} k_{A F}^{\lambda} \varepsilon_{\lambda}{ }^{\mu \rho \nu}-k_{F}^{\rho \mu \lambda \tau} R^{\nu}{ }_{\tau \rho \lambda} & m q \bar{\psi} \Gamma_{1}^{\mu} \\
-2 q \Gamma_{1}^{\nu} \psi & m\left(q \Gamma_{1}^{\rho} A_{\rho}+M_{1}-\frac{i}{2} \nabla_{\alpha} \Gamma_{1}^{\alpha}\right)+\frac{1}{2} \Gamma_{1}^{\alpha} \gamma^{\beta}\left[\nabla_{\alpha}, \nabla_{\beta}\right]
\end{array}\right)
$$

As we have already mentioned above, the operator (9) has a non-minimal form and therefore the standard Schwinger-DeWitt technique for deriving the divergences can not be applied. In this situation one can either use local momentum representation (see, e.g. 14]) or apply the generalized Schwinger-DeWitt method [15], making expansion into the new Lorentz/CPT breaking terms. The last calculation is definitely possible, in particular we could obtain the general expression for the divergences of the theory. Unfortunately, from the technical point of view, it is rather difficult, demanding extremely long and complicated algebraic computer calculations. At the same time, the most important physical information can be indeed obtained in a relatively economic way. Therefore we postpone the complete calculus for the possible next work and concentrate here on the reduced version. One can simplify the problem by setting $\hat{\mathcal{K}}^{\rho \lambda}=0$, reducing the quadratic operator to the minimal form. This restriction can be achieved by setting $k_{F}^{\mu(\rho \lambda) \nu}=0$ and $\Gamma_{1}^{\rho}=0$. In fact, $k_{F}^{\mu(\rho \lambda) \nu}$ shows up also in the expression for $\hat{L}^{\rho}$. In order to have an extra qualitative information, in what follows we will not simply set $k_{F}^{\mu(\rho \lambda) \nu}=0$, but just remember that the corresponding terms can be modified by taking into account the $\hat{\mathcal{K}}^{\rho \lambda}$-dependent terms.

In the framework of the Schwinger-DeWitt technique, we find the one-loop divergences by using the known formula (see, e.g. [10])

$$
\Gamma_{\text {div }}^{(1)}=-\frac{\mu^{\epsilon}}{(4 \pi)^{2} \epsilon} \int d^{n} x \sqrt{-g} \mathrm{~s} \operatorname{Tr}\left\{\frac{1}{2} \hat{P}^{2}+\frac{1}{12} \hat{S}_{\alpha \beta} \hat{S}^{\alpha \beta}+\text { vacuum terms }\right\},
$$

where $\epsilon=n-4$ is the dimensional regularization parameter and

$$
\begin{gathered}
\hat{P}=\hat{\Pi}+\frac{1}{6} \hat{\mathbf{1}} R-\nabla_{\mu} \hat{h}^{\mu}-\hat{h}_{\mu} \hat{h}^{\mu}, \\
\hat{S}_{\alpha \beta}=\left[\nabla_{\beta}, \nabla_{\alpha}\right] \hat{\mathbf{1}}+\nabla_{\beta} \hat{h}_{\alpha}-\nabla_{\alpha} \hat{h}_{\beta}+\hat{h}_{\beta} \hat{h}_{\alpha}-\hat{h}_{\alpha} \hat{h}_{\beta},
\end{gathered}
$$

with

$$
\hat{h}^{\rho}=\hat{h}_{0}^{\rho}+\hat{L}^{\rho} ; \quad \hat{\Pi}=\hat{\Pi}_{0}+\hat{\mathcal{M}} .
$$

Finally, the symbol sTr in the formula (14) stands for the supertrace (or Berezinian). This means that the trace of the matrix operator in (14) and in what follows is an algebraic sum of the traces of the diagonal matrix elements, with the signs taken according to the statistics of the 
corresponding quantum fields. In particular, in the bosonic sector of the fields $B^{\mu}$ the coefficient of the contribution is +1 and in the fermionic $\bar{\eta} \chi$ - sector the coefficient is -2 .

In the expression (14) we have disregarded those terms which depend only on the metric. The reason is that they are given by the sums of the contributions of the free fields which are very well-known (see, e.g. [16, 10]). After performing some algebra, we obtain, in up to the first order in the new parameters,

$$
\begin{aligned}
\frac{1}{2} \mathrm{~s} \operatorname{Tr} \hat{P}^{2} & =\frac{1}{2} \mathrm{~s} \operatorname{Tr} \hat{P}_{0}^{2}+\mathrm{s} \operatorname{Tr}\left(\hat{P}_{0} \hat{\mathcal{M}}-\hat{P}_{0} \nabla_{\nu} \hat{L}^{\nu}-\hat{P}_{0} \hat{h}_{0}^{\nu} \hat{L}_{\nu}-\hat{P}_{0} \hat{L}_{\nu} \hat{h}_{0}^{\nu}\right), \\
\mathrm{s} \operatorname{Tr} \hat{S}_{\alpha \beta} \hat{S}^{\alpha \beta} & =\mathrm{s} \operatorname{Tr}\left(\hat{S}_{0 \alpha \beta} \hat{S}_{0}^{\alpha \beta}+4 \hat{S}_{0 \alpha \beta} \nabla^{\beta} \hat{L}^{\alpha}+4 \hat{S}_{0 \alpha \beta}\left[\hat{h}_{0}^{\beta}, \hat{L}^{\alpha}\right]\right),
\end{aligned}
$$

where the operators $\hat{P}_{0}$ and $\hat{S}_{0 \alpha \beta}$ correspond to the pure QED. These operators can be obtained from the expressions (15) and (16) by setting all Lorentz/CPT breaking parameters to zero. The final result for the divergent part of the one-loop effective action has the form

$$
\begin{aligned}
\Gamma_{d i v}^{(1)} & =-\frac{\mu^{\epsilon}}{(4 \pi)^{2} \epsilon} \int d^{n} x \sqrt{-g}\left\{-2 m q H^{\mu \nu} F_{\mu \nu}-\frac{4}{3} q\left(\nabla_{\mu} a_{\nu}\right) F^{\mu \nu}\right. \\
& -2 q^{2} \bar{\psi}\left[a_{\mu} \gamma^{\mu}+\left(b_{\mu}+\frac{1}{2} S_{\mu}-3\left(k_{A F}\right)_{\mu}\right) \gamma_{5} \gamma^{\mu}+4 i m_{5} \gamma_{5}+\frac{i}{2} \nabla_{\tau}\left(k_{F}\right)^{\tau} \gamma^{\mu}\right] \psi \\
& +R_{\mu \alpha} \nabla_{\rho} \nabla_{\tau} k_{F}^{\tau \alpha \rho \mu}-\frac{1}{6} R \nabla_{\mu} \nabla_{\nu} k_{F}^{\mu \nu}+\frac{1}{3} R_{\mu \rho \alpha \beta} \nabla^{\beta} \nabla_{\tau} k_{F}^{\tau \mu \alpha \rho} \\
& \left.-\frac{1}{12} k_{F}^{\rho \lambda \mu \tau} R R_{\rho \lambda \mu \tau}+\frac{1}{2} k_{F}^{\alpha \rho \tau \lambda} R_{\rho \tau \lambda}^{\mu} R_{\mu \alpha}\right\}+\Gamma_{Q E D \text { div }}^{(1)}+\Gamma_{v a c}^{(1)}\left[g_{\mu \nu}\right],
\end{aligned}
$$

where we used notations $\left(k_{F}\right)^{\mu \lambda \nu}{ }_{\lambda}=\left(k_{F}\right)^{\mu \nu}, \quad S_{\mu}=\nabla_{\alpha} k_{F}^{\alpha \rho \lambda \sigma} \varepsilon_{\mu \rho \lambda \sigma} \quad$ and

$$
\Gamma_{Q E D \text { div }}^{(1)}=-\frac{\mu^{\epsilon}}{(4 \pi)^{2} \epsilon} \int d^{n} x \sqrt{-g}\left\{-\frac{1}{3} q^{2} F_{\mu \nu} F^{\mu \nu}+2 i q^{2} \bar{\psi}\left(\gamma^{\mu} D_{\mu}+4 i m\right) \psi\right\}
$$

is the one-loop divergences for the pure QED theory. Finally, $\Gamma_{v a c}^{(1)}\left[g_{\mu \nu}\right]$ is the divergent part of the metric-dependent vacuum effective action of QED (taking the ghosts contributions into account)

$$
\Gamma_{v a c}^{(1)}\left[g_{\mu \nu}\right]=-\frac{\mu^{\epsilon}}{(4 \pi)^{2} \epsilon} \int d^{n} x \sqrt{-g}\left\{\frac{3}{20} C_{\mu \nu \alpha \beta}^{2}-\frac{73}{360} E+\frac{2}{3} m^{2}-4 m^{4}\right\},
$$

with $C_{\mu \nu \alpha \beta}^{2}$ and $E$ representing the square of the Weyl tensor and the Gauss-Bonnet topological term (Euler characteristic). In the expressions presented above we did not include total derivatives. It is easy to see that the above expression includes, along with the usual QED divergences, many new terms. Part of these terms, concentrated in the last two lines of eq. (18) can be characterized as the vacuum ones, because they do not depend on the matter fields. Other new terms have a non-minimal form, analogous to the $R \varphi^{2}$-term for the scalar field theory in curved space. Let us notice that there is no divergent Chern-Simons - like term, $b_{\mu} A_{\nu} F_{\alpha \beta} \varepsilon^{\mu \nu \alpha \beta}$. This term is possible due to the dimensional reasons, however it does not show up in the one-loop counterterms.

\section{General discussion of the one-loop renormalization}

Let us start the analysis of the result (18) by making comparison with the available particular results. First of all, eq. (19) has the standard well-known form of the QED divergences in curved 
space-time. This is perfectly consistent with the general features of the renormalization in curved space-time (see, e.g. [10]). The renormalization of the minimal sector performs independent on external fields. We can conclude that this feature holds in the present case of a Lorentz/CPT violating theory.

As a second step, consider the particular case where the background metric is the flat Minkowski one and moreover assume that all Lorentz violation parameters are constants. This is exactly the case investigated in [8]. Unfortunately we are not able to make a full comparison, because [8] treats the general case with an arbitrary matrix $\hat{\mathcal{K}}^{\rho \lambda}$. However, even the partial comparison of the two calculations is useful and important. The one-loop divergences in this case reduce to

$$
\begin{aligned}
\Gamma_{\text {div }}^{(1)} & =\Gamma_{Q E D \text { div }}^{(1)} \\
& +\frac{2 q^{2} \mu^{\epsilon}}{(4 \pi)^{2} \epsilon} \int d^{n} x \sqrt{-g}\left\{\bar{\psi}\left[a_{\mu} \gamma^{\mu}+b_{\mu} \gamma_{5} \gamma^{\mu}+4 i m_{5} \gamma_{5}-3\left(k_{A F}\right)_{\mu} \gamma_{5} \gamma^{\mu}\right] \psi\right\}
\end{aligned}
$$

Let us notice that the term $-2 m q H^{\mu \nu} F_{\mu \nu}$ is not included here because for $H^{\mu \nu}=$ const it is a total derivative. The expression (21) corresponds to the one which has been used in [8] for deriving the renormalization group equations. Correspondingly, the proper equations (see below) reduce to the ones of [8] in the corresponding limit.

Let us now consider the qualitatively new terms due to the nontrivial metric, which have no analogs in the flat space case. The form of divergences in the fermionic sector shows the usual renormalization of the electromagnetic-like and torsion-like terms, with the $a^{\mu}$ and $b^{\mu}$ coefficients correspondingly. The form of the counterterms is controlled by the gauge symmetries, as it was discussed in [17, 18, 19]. Furthermore, the axial vector torsion component $b^{\mu}$ gains new contributions from the new $S^{\mu}$ and $k_{A F}^{\mu}$ fields. It is remarkable that the $S^{\mu}$ contribution is caused by the derivative of the massless $k_{F}^{\mu \nu \alpha \beta}$ field. Therefore if this field is present and is not exactly constant, the torsion $b^{\mu}$ term should be indeed present in the classical action, otherwise the theory is not renormalizable. Similar considerations apply to the torsion-like trace vector $a^{\mu}$. However, here we meet an interesting special situation, because the contribution from $\nabla_{\tau}\left(k_{F}\right)_{\mu}^{\tau}$ has an extra imaginary unit. In fact, this form of the imaginary term exactly corresponds to the torsion trace [19] interacting to fermion, but in the torsion case this term always cancels. Perhaps, the same concerns the corresponding quantum contributions in (18), because they may be actually cancelled by another $\nabla_{\tau}\left(k_{F}\right)_{\mu}^{\tau}$-terms coming from the $\mathcal{O}\left(\hat{\mathcal{K}}^{\rho \lambda}\right)$ contributions which we did not calculate here.

In the curvature-dependent sector the situation is also quite interesting. On dimensional reasons one could expect the counterterms of the form

$$
S_{\text {full }}=\int d^{4} x \sqrt{-g}\left\{\phi R+\phi^{\mu \nu} R_{\mu \nu}+\phi^{\mu \nu \alpha \beta} R_{\mu \nu \alpha \beta}\right\}+S_{G H D}
$$

where the last part $S_{G H D}$ represents generalized form of the higher derivative term. Let us remember that the renormalizable theory in curved space-time always includes higher derivative vacuum (metric dependent) terms (see, e.g. [16, 10]. In case when some extra field (e.g. torsion)

\footnotetext{
${ }^{5}$ At the same time, the result of [8] is restricted to the flat space, while we are working in the general curved one and also consider the non-constant Lorentz/CPT violation parameters.
} 
are present, the consistent form of the vacuum action becomes more complicated, involving the dependence of these extra fields. For example, in case of torsion the total number of possible independent structures in the vacuum action equals to 168 [20]. It is obvious that the number of necessary structures in the present case, with all new (dimensional and dimensionless) fields will be enormous and therefore we will not try to list them. Instead we just notice that, in our one-loop calculations, only two such terms emerge as counterterms and therefore

$$
S_{G H D}=\int d^{4} x \sqrt{-g}\left\{\eta_{1}^{\mu \nu \alpha \beta} R R_{\mu \nu \alpha \beta}+\eta_{2}^{\mu \nu \alpha \beta} R_{\mu}^{\lambda} R_{\lambda \nu \alpha \beta}\right\}+\ldots
$$

In the expressions (22) and (23), the coefficients $\phi, \phi^{\mu \nu}, \phi^{\mu \nu \alpha \beta}, \eta_{1}^{\mu \nu \alpha \beta}, \eta_{2}^{\mu \nu \alpha \beta}$ should depend on the new Lorentz/CPT breaking parameters in (4). The renormalizability of the theory requires that these terms should be introduced into the classical gravitational action, along with the usual Einstein-Hilbert, cosmological and higher derivative terms. Let us notice that the first term in (22) has the known metric-scalar form ${ }^{6}$, while the next two terms have a qualitatively new structure and may lead to new physical effects. Recently, an extensive investigation of the possible phenomenological manifestations of the new terms (22) has been performed in 21] on the basis of the post-Newtonian approximation. According to this work, the phenomenological manifestations of these, linear in curvature, terms are potentially measurable and therefore the theoretical status of these terms and especially their relation to the possible violation of the Lorentz/CPT symmetries in the matter sector deserve our attention. The form of divergences (18) indicates that the linear in curvatures terms do renormalize only due to the non-constant nature of the new parameters in the matter sector. Let us remark that this result does not depend on the assumption $\hat{\mathcal{K}}^{\rho \lambda}=0$ which we have used in the present work, because the $\hat{\mathcal{K}}^{\rho \lambda}$ matrix is composed by the dimensionless parameters and therefore can contribute to the dimensional coefficients $\phi, \phi^{\mu \nu}, \phi^{\mu \nu \alpha \beta}$ only through the derivatives of these parameters. All in all, the possible sources of the $\phi, \phi^{\mu \nu}$ and $\phi^{\mu \nu \alpha \beta}$ terms may be either the derivatives of the dimensionless parameters in the matter sector or the higher loop corrections. In the last case the numerical values of the contributions would be extremely weak.

\section{Renormalization group}

The renormalization group equations in the matter sector have the form which follow from the expression for the divergences

$$
\begin{aligned}
\mu \frac{d a_{\nu}}{d \mu}=\frac{d a_{\nu}}{d t}=-\frac{i q^{2}}{16 \pi^{2}} \nabla_{\alpha}\left(k_{F}\right)^{\alpha}{ }_{\nu}, & \frac{d H_{\mu \nu}}{d t}=\frac{q^{2}}{8 \pi^{2}} H_{\mu \nu}, \\
\frac{d b_{\mu}}{d t}=\frac{3 q^{2}}{8 \pi^{2}}\left(k_{A F}\right)_{\mu}-\frac{q^{2}}{16 \pi^{2}} S_{\mu}, & \frac{d k_{F}^{\mu \nu \alpha \beta}}{d t}=\frac{q^{2}}{6 \pi^{2}} k_{F}^{\mu \nu \alpha \beta}, \\
\frac{d k_{A F \mu}}{d t}=\frac{q^{2}}{6 \pi^{2}}\left(k_{A F}\right)_{\mu}, & \frac{d m_{5}}{d t}=-\frac{3 q^{2}}{8 \pi^{2}} m_{5} .
\end{aligned}
$$

\footnotetext{
${ }^{6}$ Taken alone, this action is conformally equivalent to the Brans-Dicke action and to many other versions of the metric-scalar theory. With other new terms present, this equivalence does not hold.
} 
In the particular case when all parameters are constants, these equations are exactly the ones obtained in [8]. According to the general analysis [10] these renormalizations group equations preserve their form in the curved space-time as well. The only difference is the interpretation of the renormalization group parameter $t$. The definition given in eq. (24) is the general one for the $\overline{\mathrm{MS}}$ renormalization scheme. At the same time, the standard physical interpretation in the flat space is that $t$ is the energy-momentum scaling parameter, while in curved space it is a metric scaling parameter [10] (see also further references therein). As it was already indicated in [8], the running of the parameters is small and therefore it has no much sense to analyze the renormalization group flows for these small parameters. Rather than that, the equations presented above may be used as a hint for seeing the relation between different parameters, especially for the case of non-constant ones. For instance, we observe that the vector and axial vector parts of the torsion tensor $a^{\mu}$ and $b^{\mu}$ may be caused by the variable Lorentz violating $k_{F}$ field in the vector sector.

The renormalization group equations of the new interaction parameters in the gravitational sector $\phi_{F}^{\mu \nu}, \phi, \phi^{\mu \nu}$ and $\phi^{\mu \nu \alpha \beta}$, can be written as

$$
\begin{gathered}
\frac{d \phi_{F}^{\mu \nu}}{d t}=\frac{q^{2}}{12 \pi^{2}} \phi_{F}^{\mu \nu}+\frac{m q}{8 \pi^{2}} H^{\mu \nu}+\frac{q}{12 \pi^{2}} \nabla^{\mu} a^{\nu} \\
\frac{d \phi}{d t}=\frac{1}{96 \pi^{2}} \nabla_{\mu} \nabla_{\nu} k_{F}^{\mu \nu}, \quad \frac{d \phi^{\mu \nu}}{d t}=-\frac{1}{16 \pi^{2}} \nabla_{\alpha} \nabla_{\beta} k_{F}^{\alpha \mu \beta \nu}, \quad \frac{d \phi^{\mu \nu \alpha \beta}}{d t}=-\frac{1}{48 \pi^{2}} \nabla^{\beta} \nabla_{\lambda} k_{F}^{\lambda \mu \alpha \nu} . \\
\frac{d \eta_{1}^{\mu \nu \alpha \beta}}{d t}=\frac{1}{192 \pi^{2}} k_{F}^{\mu \nu \alpha \beta}, \quad \frac{d \eta_{2}^{\mu \nu \alpha \beta}}{d t}=-\frac{1}{32 \pi^{2}} k_{F}^{\mu \nu \alpha \beta} .
\end{gathered}
$$

Here we can observe also the non-trivial nature of the variable parameters in the matter fields sector. Perhaps the most interesting is that only these variations are linked with the Lorentz violations in the gravitational sector.

\section{Conclusions}

We have investigated the one loop renormalization of the Lorentz/CPT violating QED in curved space-time, treating the Lorentz/CPT violating parameters as fields rather than as constants. The practical calculation has been performed for the "minimal" sector of the theory, admitting application of the usual Schwinger-DeWitt technique. We have found the relation between the Lorentz violating terms in the matter and gravitational sector. In particular, the corresponding gravitational terms which were recently discussed in 21], are necessary at the quantum level in case when the Lorentz violating terms in the matter section are not exactly constants.

\section{Acknowledgments.}

The authors are grateful to A. Kostelecky for stimulating discussion and correspondence. G.B.P. has been supported by the post-doctoral fellowship from PRODOC/CAPES (Brazil). I.Sh. has been partially supported by the research fellowships from $\mathrm{CNPq}$ (Brazil) and by research grants from CNPq, FAPEMIG (Minas Gerais, Brazil) and ICTP (Italy). 


\section{References}

[1] V. A. Kostelecky, S. Samuel, Phys. Rev. D39 (1989) 683;

A. Kostelecky, R. Potting, Phys. Rev. D63 (2001) 046007.

[2] R. Jackiw, A. Kostelecky, Phys. Rev. Lett. 82 (1999) 3572;

D. Colladay, A. Kostelecky, Phys.Rev. D55 (1997) 6760; Phys.Rev. D58 (1998) 116002;

A. Kostelecky, R. Lehnert, Phys. Rev. D63 (2001) 065008;

A. A. Andrianov, P. Giacconi, R. Soldati, JHEP 0202 (2002) 030; hep-th/0110279

J. Alfaro, A. A. Andrianov, M. Cambiaso, P. Giacconi and R. Soldati, Phys. Lett. B639 (2006) 586, hep-th/0604164.

[3] For the recent review and further references see

A. Kostelecky, The search for relativity violations, Sci. Am. 291 (2004) 75;

A. Kostelecky, Presented at the Third Meeting on CPT and Lorentz Symmetry, Bloomington, Indiana, August 2004, hep-ph/0412406;

R. Bluhm, Overview Of The Sme: Implications And Phenomenology Of Lorentz Violation, Talk at 339th WE Heraeus Seminar on Special Relativity, Potsdam, Germany, 13-18 Feb 2005, hep-ph/0506054;

D. Mattingly, Modern Tests Of Lorentz Invariance, Living Rev. Rel. 8 (2005) 5, gr-qc/0502097.

[4] R. Bluhm, A. Kostelecky, Phys. Rev. Lett. 84 (2000) 1381;

R. Bluhm, A. Kostelecky, C. Lane, Phys. Rev. Lett. 84 (2000) 1098.

A. Kostelecky, C. Lane, Phys. Rev. D60 (1999) 116010.

R. Bluhm, A. Kostelecky, N. Russell, Phys. Rev. Lett. 82 (1999) 2254.

[5] H. Murayama and T. Yamagida, Phys. Lett. B520 (2001) 263-268;

G. Barenboim, L. Borissov, J.D. Lykken and A. Yu Smirnov, JHEP 0210 (2002) 001;

A. Kostelecky and M. Mewes, Phys. Rev. D 70 (2004) 031902; D69 (2004) 016005.

[6] V. A. Kostelecky, Phys.Rev. D69 (2004) 105009, hep-th/0312310.

R. Bluhm, A. Kostelecky, Phys. Rev. D71 (2005) 065008, hep-th/0412320.

[7] O. Bertolami, D. Colladay, A. Kosteleck, R. Potting, Phys. Lett. B395 (1997) 178.

A. Kostelecky, M. Mewes, Phys. Rev. Lett. 87 (2001) 251304; Phys. Rev. D66 (2002) 056005.

O. Bertolami, J. Paramos, S. G. Turyshev 359th WE-Heraeus Seminar on "Lasers, Clocks, and Drag-Free: Technologies for Future Exploration in Space and Tests of Gravity," ZARM, Bremen, Germany, May-June, 2005; gr-qc/0602016. 
Ralf Lehnert, Cosmology and spacetime symmetries, (New Worlds in Astroparticle Physics, Faro, Portugal, January, 2005), hep-ph/0508316.

R. Bluhm, A. Kostelecky, Phys. Rev. D71 (2005) 065008.

[8] A. Kostelecky, C. Lane, A. Pickering, Phys.Rev. D65 (2002) 056006, hep-th/0111123;

V.Ch. Zhukovsky, A.E. Lobanov, E.M. Murchikova, Phys. Rev. D73 (2006) 065016.

[9] Manuel Perez-Victoria JHEP 0104 (2001) 032, hep-th/0102021.

[10] I.L. Buchbinder, S.D. Odintsov, I.L. Shapiro, Effective Action in Quantum Gravity (IOP Publishing, Bristol, 1992).

[11] I.L. Shapiro, J. Solà, JHEP 02 (2002) 006.

[12] B.L. Voronov, P.M. Lavrov and I.V. Tyutin, Sov.J.Nucl.Phys. 36 (1982) 498; J. Gomis and S. Weinberg, Nucl.Phys. B469 (1996) 473.

[13] S. Weinberg, The Quantum Theory of Fields: Foundations. (Cambridge Univ. Press, 1995).

[14] L. Parker and D.J. Toms, Phys. Rev. D29 (1984) 1584.

[15] A.O. Barvinsky and G.A. Vilkovisky, The generalized Schwinger-DeWitt technique in gauge theories and quantum gravity. Phys. Rep. 119 (1985) 1.

[16] N.D. Birrell and P.C.W. Davies, Quantum Fields in Curved Space (Cambridge Univ. Press, Cambridge, 1982).

[17] A.S. Belyaev, I.L. Shapiro, Phys.Lett. B425 (1998) 246; Nucl.Phys. B543 (1999) 20.

[18] G. de Berredo-Peixoto, J.A. Helayel-Neto and I. L. Shapiro, JHEP 02 (2000) 003.

[19] I.L. Shapiro, Phys. Repts. 357 (2002) 113.

[20] S.M. Christensen, J. Phys. A: Math. Gen. (1980). 133001.

[21] Q. G. Bailey and V. A. Kostelecký, Signals for Lorentz Violation in Post-Newtonian Gravity, gr-qc/0603030. 